\title{
Adiposidad corporal y bienestar psicológico: efectos de la actividad física en universitarios de Valencia, España
}

\author{
Isabel Castillo ${ }^{1}$ y Javier Molina-García ${ }^{2}$
}

Forma de citar

Castillo I, Molina-García J. Adiposidad corporal y bienestar psicológico: efectos de la actividad física en universitarios de Valencia, España. Rev Panam Salud Publica. 2009;26(4):334-40.

RESUMEN Objetivo. Determinar mediante un modelo de ecuaciones estructurales la relación que existe entre la actividad física, la adiposidad corporal, la competencia física percibida y tres indicadores del bienestar psicológico en una muestra de estudiantes universitarios españoles.

Métodos. Estudio transversal descriptivo en 639 estudiantes de 18 a 29 años representativos de las universidades de Valencia, España, en el curso 2005-2006. La práctica de actividad física se evaluó mediante el inventario de conductas de salud en escolares. Se aplicaron las escalas de competencia física percibida, autoestima, satisfacción con la vida y vitalidad subjetiva. La adiposidad corporal se expresó mediante el porcentaje de masa grasa (PMG). Se elaboró un modelo teórico con las seis variables medidas.

Resultados. El nivel de actividad física de los participantes era moderado, se percibían físicamente competentes, tenían una alta autoestima, se encontraban satisfechos con su vida y se sentían con alta vitalidad. La actividad física se relacionó negativamente con el PMG tanto en los varones como en las mujeres; el PMG se asoció negativamente con la percepción de competencia física; y la competencia física percibida se asoció positivamente con la autoestima, la satisfacción con la vida y la vitalidad subjetiva. El efecto de la práctica de actividad física sobre la competencia percibida estaba mediado parcialmente por el PMG en los varones; en las mujeres, la práctica de actividad física se relacionó directamente tanto con el PMG como con la percepción de competencia, sin la mediación del PMG.

Conclusiones. El aumento de la actividad física tiene un gran valor para la salud pública, ya que además de contribuir a tener menos grasa corporal, aumenta el bienestar psicológico y mejora la autopercepción.

Palabras clave Ejercicio; adiposidad; autoimagen; satisfacción personal; modelos psicológicos; estudiantes; España.

La práctica regular de actividad física -entendida esta como cualquier actividad que lleve al cuerpo a trabajar más de lo normal con actividades que están más

1 Departamento de Psicología Social, Facultad de Psicología, Universitat de València, España. La correspondencia se debe dirigir a Isabel Castillo, Departamento de Psicología Social, Facultad de Psicología, Universitat de València, Av. Blasco Ibáñez No. 21, CP 46010, Valencia, España. Correo electrónico: isabel.castillo@uv.es

2 Facultad de Ciencias de la Educación y del Deporte, Universidad Católica de Valencia, España. allá de la rutina diaria - se ha asociado consistentemente con diversos beneficios, tanto físicos como psicológicos, y desempeña un importante papel en la prevención de algunas enfermedades crónicas no transmisibles. Se ha demostrado que una mayor actividad física durante las primeras etapas del ciclo vital tiene efectos saludables a largo plazo, como mayor densidad ósea y resistencia cardiovascular y menor tensión arterial, sobrepeso y obesidad (1). Además, la ac- tividad física tiene efectos preventivos inmediatos contra diversas enfermedades graves, como las cardiovasculares, algunos cánceres (por ejemplo, de colon y de mama) y la diabetes mellitus tipo 2, entre otras $(2,3)$. La práctica de actividad física también se ha asociado con la disminución de síntomas de depresión, cansancio, estrés percibido y ansiedad, entre otros trastornos psicológicos (4), así como con una mayor autoestima y mejor estado de ánimo $(4,5)$. 
La prevención del sobrepeso y la obesidad constituye un objetivo prioritario de la Estrategia Mundial sobre Régimen Alimentario, Actividad Física y Salud, promovida por la Organización Mundial de la Salud y adoptada por la Asamblea Mundial de la Salud en 2004 (6). Esta iniciativa propone acciones de apoyo para la promoción de dietas saludables y la actividad física regular y pide acciones a escala mundial, regional y local dirigidas a lograr una reducción significativa de los factores de riesgo, en particular las dietas poco saludables y la inactividad física.

En las últimas décadas se han observado incrementos en la prevalencia del sobrepeso y la obesidad infantil y juvenil, más marcados en los varones y en prepúberes. En un estudio realizado en una muestra de 3534 personas de 2 a 24 años representativa de la población española, la prevalencia de sobrepeso fue $12,4 \%$ y de obesidad $13,9 \%$, en ambos casos mayor en los varones que en las mujeres (7). El incremento de la obesidad que se ha observado en muchos países es resultado de la combinación de la disminución del nivel de actividad física en la mayor parte de la población y la sobrealimentación. Estudios longitudinales y transversales indican que los jóvenes y las jóvenes con mayor actividad física tienen menores niveles de grasa corporal que los menos activos $(8,9)$. La actividad física es fundamental en el tratamiento y la prevención de la obesidad y el sobrepeso, ya que cuanto más vigorosa es, mayor es la reducción de la grasa corporal. Se considera que una persona tiene sobrepeso cuando su índice de masa corporal es mayor de 25 y que presenta adiposidad si su masa grasa corporal está por encima de $21 \%$ en los varones y de $31 \%$ en las mujeres (10).

A pesar de la elevada prevalencia de sobrepeso y obesidad en la población mundial, son pocos los estudios que han analizado sus consecuencias psicológicas (11), algo necesario para poder formular estrategias adecuadas e intervenciones más eficaces en materia de salud pública. En una muestra de 2205 niños y niñas de 9 a 10 años se encontró una relación significativa entre la adiposidad corporal y la baja competencia deportiva percibida (12). En otro estudio realizado en 2813 niños y adolescentes con una media de edad de 11 años se observó que la adiposidad corporal influyó negativamente en la autopercepción, y los chicos y las chicas obesos o con sobrepeso se percibían a sí mismos con menor competencia física en comparación con los que tenían un peso normal; los obesos tenían una autoestima significativamente más baja que sus pares de peso normal (13). A pesar de que en esos y otros estudios se ha encontrado que la adiposidad corporal puede estar relacionada con una baja percepción de la competencia física y una baja autoestima, aún hay datos contradictorios. En un estudio realizado en 2444 estudiantes de 15 a 18 años no se halló que la adiposidad tuviera un efecto directo sobre la autoestima y se sugirió que otros factores pueden mediar la relación entre la adiposidad corporal y sus consecuencias psicológicas (11). En este sentido, se ha encontrado que la autoeficacia puede desempeñar un papel mediador entre el nivel de adiposidad corporal y la depresión (14).

En cuanto a los datos empíricos sobre la relación de la adiposidad corporal con variables del bienestar psicológico, son pocos y contradictorios. En estudios realizados con adolescentes y universitarios se ha demostrado la relación existente entre la percepción de competencia física $\mathrm{y}$ diversos indicadores del bienestar psicológico, como la vitalidad subjetiva -definida como la experiencia consciente de uno mismo de poseer energía y viveza $(15,16)$ - la satisfacción con la vida - definida como una evaluación general que la persona hace sobre su vida (17) — y la autoestima —definida como la evaluación que el individuo hace y suele mantener acerca de sí mismo-, lo que lleva a una actitud favorable o desfavorable hacia el yo $(18,19)$.

De todo lo anterior se desprende que existe una relación entre la práctica regular de actividad física, el nivel de adiposidad corporal, la competencia física percibida y el bienestar psicológico. Sin embargo, no se conocen con certeza los mecanismos que producen esos beneficios, sobre todo a nivel psicológico (4), y no se ha dilucidado cómo se relacionan esas variables entre sí.

El objetivo del presente trabajo fue determinar mediante un modelo de ecuaciones estructurales la relación que existe entre la actividad física, la adiposidad corporal, la competencia física percibida y tres indicadores del bienestar psicológico (autoestima, satisfacción con la vida y vitalidad subjetiva). Por último, se discute el posible papel mediador del nivel de adiposidad corporal entre la práctica de actividad física y las variables del bienestar psicológico.

\section{MATERIALES Y MÉTODOS}

Se realizó un estudio transversal descriptivo en el que participaron 639 estudiantes universitarios representativos de los alumnos matriculados en titulaciones de primer y segundo ciclos de las universidades de Valencia, España, en el curso académico 2005-2006, con edades entre 18 y 29 años (media = 21,4; desviación estándar $=2,8$ ). De los participantes, $321(50,2 \%)$ eran varones (edad promedio: 21,7 años) y $318(49,8 \%)$ eran mujeres (edad promedio: 21,2 años).

La información se aportó de forma voluntaria y anónima entre febrero y mayo de 2006. Los cuestionarios se llenaron en aproximadamente 15 minutos y las medidas antropométricas se tomaron en cerca de 5 minutos. Al menos uno de los autores estuvo presente para dar las instrucciones previas a la aplicación de los cuestionarios y subsanar las dudas que hubieran podido surgir.

\section{Instrumentos empleados}

La práctica de actividad física se evaluó mediante la versión en español (20) del inventario de conductas de salud en escolares (21), del que se seleccionaron los ítems que miden la frecuencia, la duración y la intensidad de la actividad física. Investigaciones previas han confirmado la validez de esta medida en poblaciones universitarias (9).

Para medir la adiposidad corporal se utilizó el porcentaje de masa grasa (PMG), calculado a partir de la impedancia bioeléctrica (10) con un analizador OMRON BF $300^{\circledR}$ (Omron Matoukasa Co., Ltd., Japón). Este analizador consta de dos electrodos - uno para cada mano-y satisface los criterios de precisión por su bajo coeficiente de variación, adecuado coeficiente de correlación intraclase $(>0,95)$ y buenos parámetros de validación (error técnico excelente, diferencias clínicamente aceptables y coeficiente de correlación intraclase $>0,75$ ) (22). Diversos estudios epidemiológicos han mostrado que este analizador es una herramienta confiable para la medición del porcentaje de grasa corporal (23).

La competencia se evaluó mediante la versión en español (24) de la escala de competencia percibida del cuestionario de motivación intrínseca (25). Esta escala 
está compuesta por cinco ítems que evalúan la competencia física percibida. Se solicitó a los participantes que se ubicaran en un nivel de competencia de acuerdo con una serie de afirmaciones como "Creo que soy bastante bueno/a en mi actividad físico-deportiva". Las respuestas se dieron en una escala de tipo Likert que iba desde 1 (totalmente en desacuerdo) hasta 7 (totalmente de acuerdo). Investigaciones previas han confirmado la confiabilidad de esta escala (24).

Para medir la autoestima se utilizó la versión en español (26) de la escala de autoestima (16). Esta escala permite evaluar la autoestima de manera general mediante 10 ítems que exploran los sentimientos generales acerca del yo. Diversas investigaciones han mostrado una adecuada consistencia interna de este instrumento (27). Las valoraciones personales a afirmaciones como "a veces pienso que no sirvo para nada" se dieron en una escala de tipo Likert que iba desde 1 (muy en desacuerdo) hasta 4 (muy de acuerdo).

La satisfacción con la vida se evaluó mediante la versión en español (28) de una escala específica para este fin (45). Esta escala de cinco ítems ha mostrado una adecuada consistencia interna (28, 29). Los participantes indicaron su grado de acuerdo con afirmaciones como "en la mayoría de los aspectos, mi vida es como yo quiero que sea" mediante una escala de tipo Likert que iba desde 1 (muy en desacuerdo) hasta 7 (muy de acuerdo).

Para evaluar la vitalidad se utilizó la versión en español ${ }^{3}$ de la escala de vitalidad subjetiva (15). Esta escala de siete ítems mide los sentimientos subjetivos generales de viveza y energía. Se le pidió a los participantes que indicaran el grado en que, por lo general, una serie de afirmaciones eran verdaderas para ellos (por ejemplo, "me siento vivo y vital"). Las respuestas se dieron según una escala de tipo Likert que iba desde 1 (no es verdad) hasta 7 (verdadero). Esta escala ha mostrado una adecuada consistencia interna (15).

Siguiendo la experiencia de investigaciones previas $(9,30)$, el nivel de activi-

\footnotetext{
Balaguer I, Castillo I, García-Merita M, Mars L. Implications of structured extracurricular activities on adolescent's well being and risk behaviors: motivational mechanisms [oral presentation]. 9th European Congress of Psychology; Granada, Spain; 3-8 July, 2005.
}

dad física se clasificó en este estudio en cinco niveles según su frecuencia, intensidad y duración: 1: sedentario (nunca practica actividad física); 2: bajo (practica no más de una vez por semana en sesiones de 30 minutos o más); 3: moderado (practica 2-3 veces por semana en sesiones de 30 minutos o más); 4: alto (practica 4-5 veces por semana en sesiones de 30 minutos o más); y 5: muy alto (practica 6-7 veces por semana en sesiones de 30 minutos o más).

Se examinó la estructura factorial de las escalas utilizadas en el estudio mediante el análisis factorial confirmatorio. Los resultados mostraron que todas las escalas poseían índices de ajuste satisfactorios, así como saturaciones factoriales adecuadas. (Por razones de brevedad no se presentan los resultados del análisis factorial confirmatorio, pero están disponibles y pueden solicitarse a los autores.)

Se elaboró un modelo teórico con seis variables observables: práctica de actividad física, PMG, competencia física percibida, autoestima, satisfacción con la vida y vitalidad subjetiva. Este modelo se probó de manera independiente para cada sexo con la técnica de análisis de vías causales (path analysis) mediante el método de máxima verosimilitud del programa LISREL 8.54 (31).

Se emplearon diversos índices de ajuste: la relación entre el valor de la ji al cuadrado y los grados de libertad $\left(\chi^{2} / \mathrm{gl}\right)$, el índice de ajuste no normativo (IANN), el índice de ajuste comparativo (IAC) y la raíz media cuadrática del error de aproximación (RMCEA). Una relación $\chi^{2} / \mathrm{gl}<3$ indica un buen ajuste del modelo y valores del IAC y del IANN mayores de 0,90 indican un ajuste aceptable. El valor de la RMCEA debe ser menor que 0,08 , aunque se considera aceptable si está entre 0,05 y 0,10 (32).

\section{RESULTADOS}

La actividad física de los estudiantes universitarios valencianos participantes en este estudio estaba en un nivel moderado, se percibían físicamente competentes, informaron tener una alta autoestima, se encontraban satisfechos con sus respectivas vidas y se sentían con alta vitalidad (cuadro 1). El PMG, tanto en los varones como en las mujeres, estaba en los niveles normales. Los varones practicaban más actividad física que las mujeres, se percibían con mayor competencia física y autoestima y se sentían más vita- les que sus pares mujeres; estas, por su parte, tenían un mayor PMG que los varones $(P<0,05)$.

El análisis de correlación confirmó en ambos sexos una relación negativa entre la actividad física y el PMG, y positiva entre la actividad física y la percepción de competencia; la percepción de competencia se relacionó positivamente con las variables del bienestar psicológico. El PMG se relacionó negativamente tanto con la percepción de competencia como con las variables del bienestar psicológico en los varones; pero estas relaciones no resultaron significativas en las mujeres.

A juzgar por los valores del coeficiente alfa de Cronbach, en este estudio se observó una adecuada fiabilidad de la escala de competencia física percibida (varones: 0,77; mujeres: 0,75), la de autoestima (varones: 0,81; mujeres: 0,82), la de satisfacción con la vida (varones: 0,84 ; mujeres: 0,89$)$ y la de vitalidad subjetiva (varones: 0,85; mujeres: 0,90).

\section{Análisis de las vías causales}

El modelo teórico ofreció un ajuste adecuado de los datos, tanto en los varones $\left(\chi^{2}[7]=23,76, P<0,01 ; \chi^{2} / \mathrm{gl}=3,39\right.$; $\mathrm{RMCEA}=0,06$; IANN $=0,91 ; \mathrm{IAC}=0,93$ ) como en las mujeres $\left(\chi^{2}[7]=26,06\right.$, $P<0,01 ; \chi^{2} / \mathrm{gl}=3,72 ; \mathrm{RMCEA}=0,07$; IANN $=0,90$; IAC $=0,92$ ). Los parámetros de la solución estandarizada muestran que la práctica de actividad física se relacionó negativamente con el PMG, tanto en los varones $(\beta=-0,35)$ como en las mujeres $(\beta=-0,32)$. Asimismo el PMG se asoció negativamente con la percepción de competencia física $(\beta=-0,24$ y $\beta=-0,13$, respectivamente).

Por su parte, en los varones como en las mujeres, la competencia física percibida se asoció positivamente con la autoestima $(\beta=0,43$ y $\beta=0,18$, respectivamente), la satisfacción con la vida ( $\beta=0,28$ y $\beta=0,20$, respectivamente) y la vitalidad subjetiva $(\beta=0,30$ y $\beta=0,30$, respectivamente) (figura 1 ).

\section{Análisis de la mediación}

Según las recomendaciones de Holmbeck (33), se probaron varios modelos alternativos para determinar si el PMG mediaba el efecto de la práctica de actividad física sobre la percepción de competencia y se aplicó la prueba de Sobel para establecer el significado de esa mediación (34). 
CUADRO 1. Comportamiento de las variables estudiadas y sus correlaciones

\begin{tabular}{|c|c|c|c|c|c|c|c|c|c|c|c|c|c|c|}
\hline \multirow[b]{2}{*}{ Variable } & \multirow{2}{*}{$\begin{array}{l}\text { Posibles } \\
\text { valores } \\
\text { (mínimo- } \\
\text { máximo) }\end{array}$} & \multicolumn{2}{|c|}{ Total } & \multicolumn{2}{|c|}{ Varones } & \multicolumn{2}{|c|}{ Mujeres } & \multirow[b]{2}{*}{$t^{\mathrm{a}}$} & \multicolumn{6}{|c|}{ Correlación entre las variables ${ }^{b}$} \\
\hline & & Media & $\mathrm{DE}^{\mathrm{c}}$ & Media & DE & Media & $\mathrm{DE}$ & & 1 & 2 & 3 & 4 & 5 & 6 \\
\hline $\begin{array}{l}\text { 1. Práctica de } \\
\text { actividad física } \\
\text { 2. Porcentaje de }\end{array}$ & $1-5$ & 2,73 & 1,19 & 3,26 & 0,96 & 2,18 & 1,16 & 12,58 & - & $-0,32^{d}$ & $0,23^{d}$ & 0,01 & 0,00 & $0,21^{d}$ \\
\hline $\begin{array}{l}\text { masa grasa } \\
\text { 3. Competencia }\end{array}$ & $6,2-49,3$ & 24,68 & 8,24 & 18,75 & 5,36 & 30,67 & 6,01 & 26,47 & $-0,35^{d}$ & - & $-0,13$ & $-0,01$ & $-0,04$ & $-0,07$ \\
\hline percibida & $1-7$ & 5,28 & 0,84 & 5,34 & 0,84 & 5,18 & 0,84 & 2,08 & $0,31^{d}$ & $-0,24^{d}$ & - & $0,18^{d}$ & $0,20^{d}$ & $0,29^{d}$ \\
\hline $\begin{array}{l}\text { 4. Autoestima } \\
\text { 5. Satisfacción }\end{array}$ & $1-4$ & 3,15 & 0,45 & 3,23 & 0,44 & 3,07 & 0,44 & 4,59 & 0,11 & $-0,17^{d}$ & $0,43^{d}$ & - & $0,53^{d}$ & $0,46^{d}$ \\
\hline $\begin{array}{l}\text { con la vida } \\
\text { 6. Vitalidad }\end{array}$ & $1-7$ & 5,33 & 1,07 & 5,40 & 0,97 & 5,26 & 1,17 & 1,63 & 0,07 & $-0,14^{d}$ & $0,28^{d}$ & $0,43^{d}$ & - & $0,52^{d}$ \\
\hline subjetiva & $1-7$ & 4,94 & 1,09 & 5,12 & 0,99 & 4,75 & 1,15 & 4,31 & $0,24^{d}$ & $-0,17^{d}$ & $0,30^{d}$ & $0,42^{\mathrm{d}}$ & $0,41^{d}$ & - \\
\hline
\end{tabular}

a Prueba $t$ de Student entre varones y mujeres. Valores de $t>1,96$ son estadísticamente significativos $(P<0,05)$.

${ }^{b}$ Coeficientes de correlación de Pearson $(r)$. Los valores ubicados por encima de la diagonal en blanco corresponden a las mujeres y los ubicados por debajo a los varones.

${ }^{c} \mathrm{DE}$ : desviación estándar.

d Correlación significativa $(P<0,05)$.

En un primer modelo teórico, que exploraba la relación directa entre la actividad física y la percepción de competencia —que a su vez se relaciona con las variables del bienestar psicológico-, el ajuste de los datos fue adecuado tanto en los varones $\left(\chi^{2}[3]=7,68, P>0,01\right.$; $\mathrm{RMCEA}=0,03$; IANN $=0,96$; IAC $=0,97$ ) como en las mujeres $\left(\chi^{2}[3]=16,18\right.$, $P<0,01 ;$ RMCEA $=0,04 ;$ IANN $=0,94 ;$ IAC $=0,94)$. En este modelo, el coeficiente de regresión fue positivo y significativo entre la práctica de actividad física y la percepción de competencia tanto en los varones como en las mujeres ( $\beta=0,31$ y $\beta=0,23$, respectivamente; $P<0,001)$. Estos resultados se examinaron en un segundo modelo, restringido, que mostró un ajuste adecuado de los datos y el signo de los coeficientes fue en el sentido previsto (figura 1).
Al analizar más adelante un tercer modelo no restringido -igual al anterior, pero en el que se evaluó la posible relación directa entre la práctica de actividad física y la percepción de competenciase observó también un ajuste adecuado de los datos, tanto en los varones $\left(\chi^{2}[6]=\right.$ $8,01, P>0,01$; RMCEA $=0,04$; IANN = $0,96$; IAC $=0,98)$ como en las mujeres $\left(\chi^{2}[6]=16,10, P<0,01 ;\right.$ RMCEA = 0,04; IANN $=0,94 ;$ IAC $=0,95)$. El coeficiente de regresión directo entre la práctica de actividad física y la competencia percibida resultó significativo en los varones y las mujeres $(\beta=0,26$ y $\beta=0,21$, respectivamente; $P<0$,001) (figura 2). Al comparar estos resultados con los del segundo modelo (restringido) se observó una reducción en el valor del coeficiente de regresión estandarizado (coeficiente $\beta$ ) entre el PMG y la competencia percibida en el grupo de los varones $(\beta=-0,24$ frente a $\beta=-0,15 ; P<0,001)$ y se redujo el nivel de significación estadística en el grupo de las mujeres $(\beta=-0,13 ; P<0,001$ frente a $\beta=-0,06 ; P>0,01$ ); en el resto de los coeficientes de regresión se mantuvieron los mismos valores que en el modelo restringido.

El análisis de la diferencia de los valores de la ji al cuadrado $\left(\Delta \chi^{2}\right)$ mostró valores significativos en el segundo y el tercer modelo, tanto en los varones $\left(\Delta \chi^{2}[1]=15,75 ; P<0,01\right)$ como en las mujeres $\left(\Delta \chi^{2}[1]=9,96 ; P<0,01\right)$, lo que indica que la relación directa entre la práctica de actividad física y la competencia percibida se ajustaba mejor en el tercer modelo (figura 2) que en el restringido (figura 1). Esto llevaría a refutar la hipótesis de una mediación total del PMG. No obstante, un análisis más pormenori-

FIGURA 1. Resultados del análisis de vías causales en el modelo de ecuaciones estructurales ${ }^{a}$

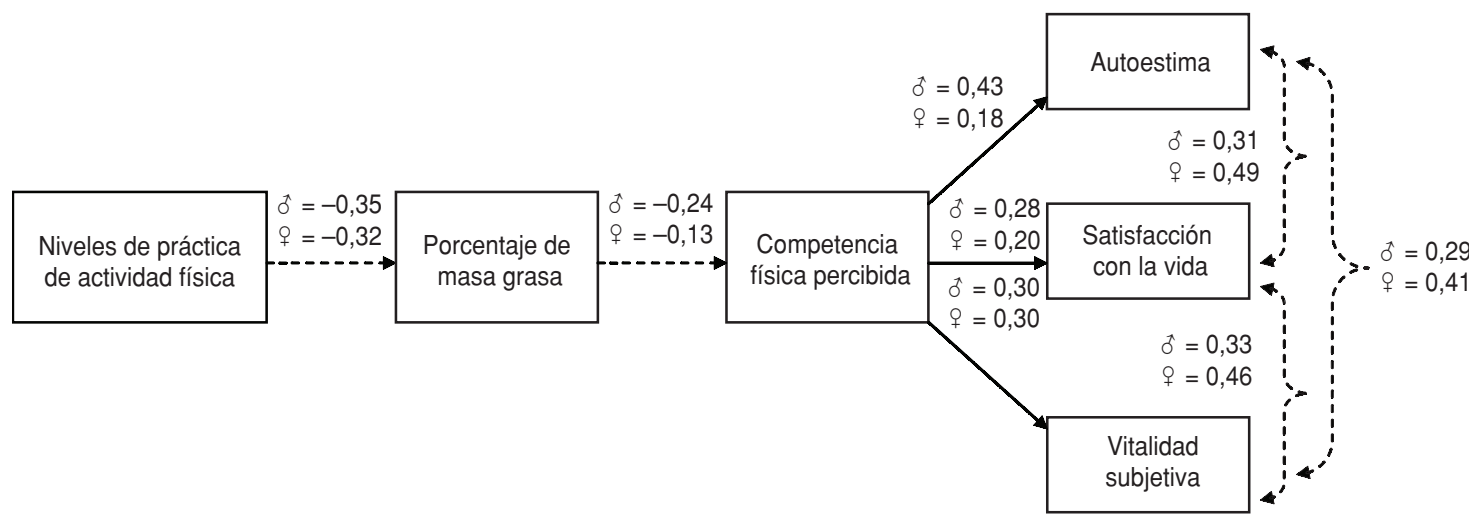

${ }^{a}$ Las cifras indican el valor del coeficiente de regresión estandarizado (coeficiente $\beta$ ). Todas las variables de la regresión se expresan en forma estandarizada o tipificada (puntuaciones $z$ ). Nivel de significación $P<0,05$. 
FIGURA 2. Resultados del análisis de mediación en el modelo de ecuaciones estructurales ${ }^{a}$

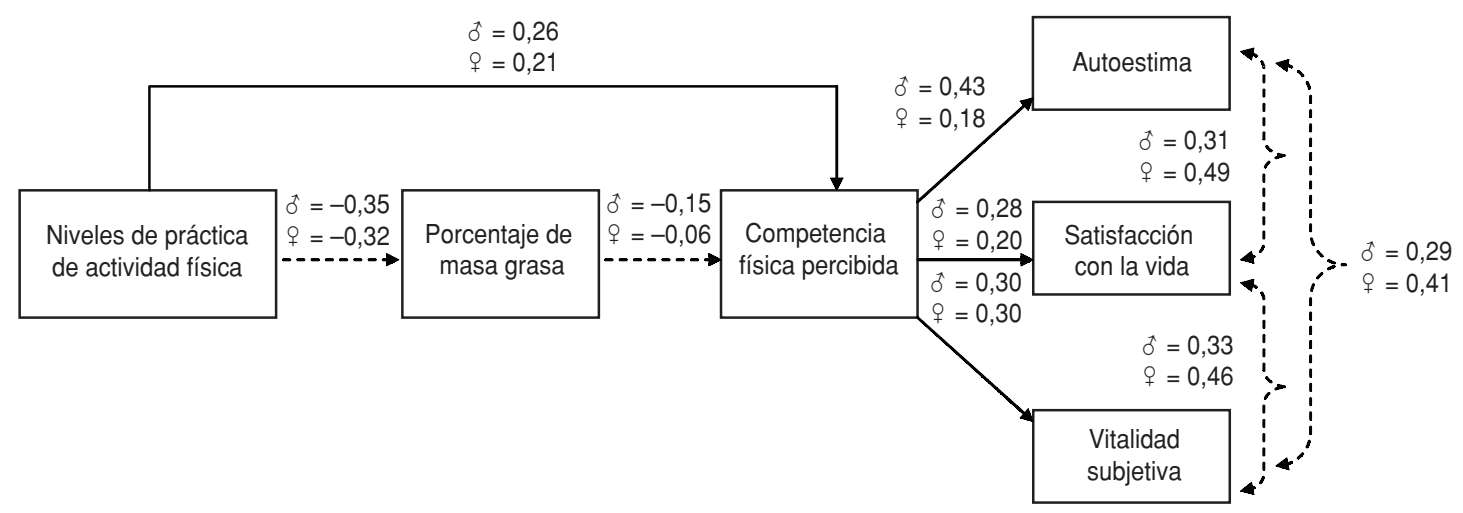

a Las cifras indican el valor del coeficiente de regresión estandarizado (coeficiente $\beta$ ). Todas las variables de la regresión se expresan en forma estandarizada o tipificada (puntuaciones $z$ ). Puntuaciones igual a 0,15 o mayores son significativas $(P<0,05)$.

zado de los coeficientes de regresión mostró que había una mediación parcial, ya que si bien el efecto directo entre la práctica de actividad física y la competencia percibida seguía siendo estadísticamente significativo cuando mediaba el efecto del PMG, el coeficiente beta era menor que cuando no mediaba este efecto (en los varones el efecto se redujo de $\beta=0,31$ a $\beta=0,26, P<0,001$; en las mujeres se redujo de $\beta=0,23$ a $\beta=0,21$, $P<0,001)$. Los resultados de la prueba de Sobel mostraron el efecto mediador del PMG entre la práctica de actividad física y la competencia percibida, significativo en los varones $(z=1,91 ; P<0,05)$, pero no significativo en las mujeres ( $z=0,99 ; P>0,05)$.

En resumen, el análisis de la mediación confirmó que el efecto de la práctica de actividad física sobre la competencia percibida estaba mediado por el PMG en los varones, aunque esta mediación fue parcial. En otras palabras, en los varones la práctica de actividad física se relaciona tanto directa como indirectamente - a través del PMG - con la percepción de competencia (figura 2). En cuanto a las mujeres, la práctica de actividad física se relacionó directamente tanto con el PMG como con la percepción de competencia, sin la mediación del PMG (figura 2).

\section{DISCUSIÓN}

El modelo de ecuaciones estructurales empleado en una muestra representativa de estudiantes universitarios de Valencia, España, permitió establecer una relación directa entre la práctica de actividad física, tres indicadores del bienestar psicológico (autoestima, satisfacción con la vida y vitalidad subjetiva) - mediados por el PMG - y la competencia física percibida.

Los resultados confirman que la práctica de actividad física está inversamente relacionada con el nivel de adiposidad corporal y estaría en consonancia con un estudio longitudinal realizado recientemente en adolescentes de 12 a 17 años en el que se encontró que la actividad física regular (más de dos veces a la semana) ayudó a prevenir el sobrepeso en la edad adulta (35).

En el presente estudio, el PMG se asoció negativamente con la percepción de competencia en ambos sexos. Además, la competencia percibida se asoció positivamente con la autoestima, la satisfacción con la vida y la vitalidad subjetiva de los participantes en el presente estudio. Esto podría indicar que el sentirse competentes lleva a los jóvenes a tener una visión más favorable acerca de sí mismos, a evaluarse más positivamente y a sentirse con más energía y viveza.

Los jóvenes hacen juicios y evaluaciones sobre sí mismos. Estas evaluaciones pueden llevarlos a sentimientos positivos o negativos respecto a sus percepciones de competencia, lo cual a su vez influye en su bienestar psicológico, motivación, interés y persistencia en una tarea (36). La inactividad física es un factor que contribuye al sobrepeso y a la obesidad (37) y el sobrepeso y la obesidad, a su vez, reducen la motivación a involucrarse en actividades físicas. Además, el sobrepeso y la obesidad se aso- cian con una baja percepción de competencia, y esta baja autopercepción afecta directamente a otras variables del bienestar psicológico.

Es importante entender los mecanismos que se relacionan con las percepciones de competencia por sus implicaciones para la salud pública y su utilidad para diseñar y aplicar programas eficaces que promuevan la práctica de actividad física. Esto es de especial importancia en las mujeres, ya que por lo general son menos activas físicamente y se perciben con peor competencia física que los varones (38). Varios autores han mostrado que las mujeres pueden beneficiarse más que los hombres, tanto física como psicológicamente, de la práctica de actividad física $(39,40)$.

Un aspecto importante de este estudio es el análisis del papel mediador de la adiposidad corporal en la relación entre la práctica de actividad física y la autopercepción y el bienestar psicológico de los jóvenes. Según el análisis de la mediación, la práctica de actividad física tiene en los varones un efecto significativo sobre la percepción de competencia, mediado por el PMG. En otras palabras, los universitarios físicamente activos poseen un menor PMG, lo que hace que se perciban a sí mismos más competentes. Los varones ven mejorada esta percepción de competencia, tanto mediante la actividad física como por su menor PMG. Sin embargo, las universitarias que practican actividad física reducen su PMG y mejoran su percepción de competencia, pero su PMG no tiene un efecto significativo sobre cuán competentes físicamente se 
sienten. Estos resultados confirman empíricamente las diferencias de género en cuanto a los efectos de la práctica regular de actividad física (38).

La adiposidad corporal en las mujeres del presente estudio no se asoció con su percepción de competencia y bienestar psicológico. Sin embargo, se encontró que con la práctica de actividad física se redujo su PMG y aumentó su percepción de competencia, lo que provocó un mayor bienestar psicológico. Los aspectos culturales pueden explicar en parte este hallazgo, ya que el estar físicamente activos o percibirse físicamente competentes puede verse como un valor del estereotipo masculino; mientras que el sobrepeso y la baja percepción de competencia física se asocian más con el estereotipo femenino. Esta hipótesis de que el sobrepeso se asocia con lo femenino podría también explicar que las estudiantes universitarias de la muestra no relacionen significativamente su PMG con los diferentes indicadores del bienestar psicológico, como se observó en los varones. Otra posible explicación podría ser que, al contrario de lo que ocurriría en los va- rones - en los que la construcción de su autopercepción se basa en lo físico y un aumento del PMG se asocia con un menor bienestar psicológico-, las mujeres no construyen su autopercepción a partir de su aptitud física, de ahí que el PMG y la percepción de su aptitud y competencia físicas sean constructos independientes. Futuras investigaciones deberán profundizar en el estudio cualitativo del contenido cultural y socializador de estas diferencias de género.

El presente estudio tiene algunas limitaciones que se deben tomar en cuenta. En primer lugar, su naturaleza transversal impide hacer asociaciones de causa y efecto. No obstante, los modelos de ecuaciones estructurales permitieron establecer relaciones lineales entre los constructos, lo que podría sugerir conexiones causales. Mediante el análisis de vías causales se evaluaron las influencias directa e indirecta de la práctica de actividad física - considerada como causasobre otras variables - tomadas como efectos-, bajo el presupuesto de que existen relaciones lineales entre la primera y el resto de las variables, que los errores del modelo de regresión no están correlacionados y que no hay errores sistemáticos de medición de las variables (32). En segundo lugar, se utilizó una muestra representativa de la población de estudiantes universitarios de Valencia, España, por lo que en rigor, los resultados solo podrían generalizarse a este segmento de la población.

A pesar de esas limitaciones, los resultados de este estudio confirman que el aumento de la actividad física tiene un gran valor desde el punto de vista de la salud pública, ya que además de contribuir a tener menos grasa corporal, aumenta el bienestar psicológico y mejora la autopercepción.

Se debe estudiar el papel modulador de la edad en relación con el peso corporal, ya que existe una tendencia generalizada a un aumento en la prevalencia de obesidad infantil y juvenil y al incremento del peso con la edad. La realización de estudios longitudinales podría contribuir a comprender mejor los mecanismos mediante los cuales la actividad física permite mejorar tanto el estado físico como el bienestar psicológico.

\section{REFERENCIAS}

1. U S Department of Health and Human Service. Physical activity and health: a report of the Surgeon General. Atlanta, GA: USDHHS, Centers for Disease Control and Prevention, National Center for Chronic Disease Prevention and Health Promotion; 2002.

2. World Health Organization. Information sheets: physical activity. Geneva: WHO; 2003. Hallado en http://www.who.int/dietphysi calactivity/media/en/gsfs_pa.pdf. Acceso el 15 de agosto de 2009.

3. World Health Organization. Joint WHO/ FAO expert consultation on diet, nutrition, and the prevention of chronic diseases, 2002. Geneva: WHO; 2003. (Technical Report Series No. 916.)

4. Jiménez MG, Martínez P, Miró E, Sánchez AI. Bienestar psicológico y hábitos saludables: ¿están asociados a la práctica de ejercicio físico? Int J Clin Health Psychol. 2008;8: 185-202.

5. Biddle SJH, Fox KR, Boutcher SH. Physical activity and psychological wellbeing. London: Routledge; 2000.

6. World Health Organization. Global Strategy on Diet, Physical Activity and Health. Geneva: WHO; 2004.

7. Serra Majem L, Ribas Barba L, Aranceta Bartrina J, Pérez Rodrigo C, Saavedra Santana P, Peña Quintana L. Obesidad infantil y juvenil en España. Resultados del Estudio enKid (1998-2000). Med Clin (Barcelona). 2003;121: $725-32$.
8. Barbeau P, Litaker MS. Effect of a physical activity intervention on bone mineral density in black girls: relative contribution of changes in fat mass and fat free soft tissue. Med Sci Sports Exerc. 2003;35:S82.

9. Molina-García J, Castillo I, Pablos C, Queralt A. La práctica de deporte y la adiposidad corporal en una muestra de universitarios. Apunts Educ Fis Deportes. 2007;89:23-30.

10. Sociedad Española para el Estudio de la Obesidad. Consenso SEEDO 2000 para la evaluación del sobrepeso y la obesidad y el establecimiento de criterios de intervención terapéutica. Med Clin (Barcelona). 2000;115: 587-97.

11. Ozmen D, Ozmen E, Ergin D, Cetinkaya AC, Sen N, Dundar PE, et al. The association of self-esteem, depression and body satisfaction with obesity among Turkish adolescents. BMC Public Health. 2007;7:80.

12. Kimm SY, Barton BA, Berhane K, Ross JW, Payne GH, Schreiber GB. Self-esteem and adiposity in black and white girls: the NHLBI Growth and Health Study. Ann Epidemiol. 1997;7:550-60.

13. Franklin J, Denyer G, Steinbeck KS, Caterson IA, Hill AJ. Obesity and risk of low selfesteem: a statewide survey of Australian children. Pediatrics. 2006;118:2481-7.

14. Sacco WP, Wells KJ, Friedman A, Matthew R, Perez S, Vaughan CA. Adherence, body mass index, and depression in adults with type 2 diabetes: the mediational role of diabetes symptoms and self-efficacy. Health Psicol. 2007;26:693-700.

15. Ryan RM, Frederick CM. On energy, personality, and health: subjective vitality as a dynamic reflection of well-being. J Pers. 1997;65: 529-65.

16. Reinboth M, Duda JL, Ntoumanis N. Dimensions of coaching behavior, need satisfaction, and the psychological and physical welfare of young athletes. Motiv Emotion. 2004;28: 297-313.

17. Pavot W, Diener E, Colvin CR, Sandvik E. Further validation of the satisfaction with life scale: evidence for the cross-method convergence of well-being. Soc Indic Res. 1991;28:1-20.

18. Rosenberg M. Society and the adolescent selfimage. Princeton, NJ: Princeton University Press; 1965.

19. Reinboth M, Duda JL. The motivational climate, perceived ability, and athletes' psychological and physical well-being. Sport Psychol. 2004;18:237-51.

20. Balaguer I. Estilos de vida en la adolescencia. Valencia: Promolibro; 2002.

21. Wold B. Health-behavior in schoolchildren: a WHO cross-national survey. Resource Package Questions 1993-94. Bergen: University of Bergen; 1995.

22. Martín V, Gómez B, Antoranz MJ, Fernández S, Gómez A, de Oya M. Validación del monitor de medición de la grasa corporal por impedancia bioeléctrica OMRON BF 300. Aten Primaria. 2001;28:174-81. 
23. Lintsi M, Kaarma H, Kull I. Comparison of hand-to-hand bioimpedance and anthropometry equations versus dual-energy $\mathrm{X}$-ray absorptiometry for the assessment of body fat percentage in 17-18-year-old conscripts. Clin Physiol Funct Imaging. 2004;24:85-90.

24. Balaguer I, Castillo I, Duda JL. Apoyo a la autonomía, satisfacción de las necesidades, motivación y bienestar en deportistas de competición: un análisis de la teoría de la autodeterminación. Rev Psicol Deporte. 2008; 17(1):123-39.

25. McAuley E, Duncan T, Tammen VV. Psychometric properties of the intrinsic motivation inventory in a competitive sport setting: a confirmatory factor analysis. Res Q Exerc Sport. 1989;60:48-58.

26. Atienza FL, Moreno Y, Balaguer I. Análisis de la dimensionalidad de la escala de autoestima de Rosenberg en una muestra de adolescentes valencianos. Rev Psicol Universitas Tarraconensis. 2000;22:29-42.

27. Gotwals J, Wayment HA. Evaluation strategies, self-esteem and athletic performance. Curr Res Soc Psychol. 2002;8:84-101.

28. Atienza FL, Pons D, Balaguer I, García-Merita M. Propiedades psicométricas de la escala de satisfacción con la vida en adolescentes. Psicothema. 2000;12:314-9.

29. Diener E, Emmons R, Larsen RJ, Griffin S. The satisfaction with life scale. J Pers Assess. 1985;49:71-5.

30. Pastor Y, Balaguer I, García-Merita M. Estilo de vida y salud. Valencia: Albatros; 1999.

31. Jöreskog K, Sörbom D. LISREL 8.54. Structural equation modeling with the SIMPLIS command language. Chicago: Scientific Software International; 2003.

32. Hoyle RH. Structural equation modeling: concepts, issues and applications. Thousand Oaks, CA: Sage; 1995.

33. Holmbeck GN. Toward terminological, conceptual, and statistical clarity in the study of mediators and moderators: examples from the child-clinical and pediatric psychology literatures. J Consult Clin Psychol. 1997:65:599-610.

34. Sobel ME. Asymptotic intervals for indirect effects in structural equations models. In: Leinhart S, ed. Sociological methodology. San Francisco, CA: Jossey-Bass; 1982. Pp. 290-312.

35. Menschik D, Ahmed S, Alexander MH, Blue RW. Adolescent physical activities as predictors of young adult weight. Arch Pediatr Adolesc Med. 2008;162:29-33.
36. Feltz DL, Petlichkoff L. Perceived competence among interscholastic sport participants and dropouts. Can J Appl Sport Sci. 1983;8:231-5.

37. Trost SG, Kerr LM, Ward DS, Pate RR. Physical activity and determinants of physical activity in obese and non-obese children. Int Obes. 2001;25:822-9.

38. Castillo I, Balaguer I, García-Merita M. Efecto de la práctica de actividad física y de la participación deportiva sobre el estilo de vida saludable en la adolescencia en función del género. Rev Psicol Deporte. 2007;16:201-10.

39. Kull M. The relationships between physical activity, health status and psychological wellbeing of fertility-aged women. Scand J Med Sci Sports. 2002;12:241-7.

40. Stein C, Fisher L, Berkey C, Colditz G. Adolescent physical activity and perceived competence: does change in activity level impact self-perception? J Adolesc Health. 2007;40: $462-70$.

Manuscrito recibido el 23 de abril de 2008. Aceptado para publicación, tras revisión, el 1 de noviembre de 2008.
ABSTRACT

\section{Adiposity and psychological well-being: effects of physical activity on university students in Valencia, Spain}

Key words
Objective. To determine, through the use of a structural equation model, the relationships that exist between physical activity, body fat, perceived physical ability, and three indicators of psychological well-being, in a sample of Spanish university students.

Methods. A descriptive cross-sectional study of 639 students 18-29 years of age representative of the universities of Valencia, Spain, during the 2005-2006 term. Physical exercise was rated by taking an inventory of healthy behaviors among students. The following scales were applied: self-perceived physical ability, self-esteem, satisfaction with life, and subjective vitality. Body fat was expressed as the percentage of fat mass (PFM). A theoretical model was devised using six measured variables.

Results. The participants' level of physical activity was moderate; they perceived themselves to be physically competent; had high self-esteem; were satisfied with life; and had high vitality. Physical activity was negatively correlated with PFM in men and women alike; and negatively associated with perceived physical ability; while perceived physical ability was positively associated with self-esteem, life satisfaction, and subjective vitality. The effect of physical activity on perceived competence was mediated in part by PFM in men. In women, exercise was directly correlated to PFM, as well as perceived ability, without PFM mediation.

Conclusions. Increased physical activity is of great value to public health because, in addition to helping to reduce body fat, it improves psychological well-being and self-image.

Exercise; adiposity; self-concept; personal satisfaction; models, psychological; students; Spain. 